\title{
Application of the phenomenological renormalization to percolation and lattice animals in dimension 2
}

\author{
B. Derrida and L. De Seze $\left(^{*}\right)$ \\ Service de Physique Théorique, CEN Saclay, 91191 Gif sur Yvette Cedex, France
}

(Reçu le 28 septembre 1981, accepté le 12 novembre 1981)

\begin{abstract}
Résumé. - Après avoir rappelé la relation entre les lois d'échelles pour les systèmes finis et la renormalisation phénoménologique nous calculons l'exposant $v$ pour la percolation en dimension 2 et trouvons un bon accord avec la valeur de $4 / 3$ proposée par den Nijs. Nous avons construit les matrices de transfert pour le problème des animaux afin de calculer les longueurs de corrélation. Nous trouvons $v=0,6408 \pm 0,0003$ en dimension 2 .
\end{abstract}

\begin{abstract}
We recall the relation between finite-size scaling and the phenomenological renormalization. We calculate the exponent $v$ in dimension 2 for percolation and find a good agreement with the conjecture $4 / 3$ of den Nijs. For lattice animals, we construct transfer matrices to calculate the correlation lengths and we find
\end{abstract}

$$
v=0.6408 \pm 0.0003
$$

in dimension 2.

1. Introduction. - Since Nightingale [1] introduced the phenomenological renormalization (PR) method in the study of the Ising model, many authors used this approach to calculate the critical behaviour of other models like generalized Ising models [2], Ising antiferromagnets in a magnetic field [3, 4], lattice gas models [4], quantum spin systems [5], Lee and Yang singularities [6]. For all these models, the transfer matrix or the hamiltonian can be written obviously. In order to apply the PR method to percolation, it was necessary to show how the idea of transfer matrix could be generalized [7]. Subsequently, other geometrical problems like the self avoiding walk [8] or directed percolation [9] were studied by the PR method. The first motivation of the present work was to continue the work of Derrida and Vannimenus [7] and to find the best prediction of the PR method for the exponent $v$ of percolation. Once our programs of constructing the transfer matrices for percolation were reliable, it became very easy to study also the problem of lattice animals.

The literature on percolation is considerable : for recent reviews on the field, we refer to the course of Kirkpatrick [10] and to the articles of Stauffer [11]

(*) Service de Physique du Solide et de Résonance Magnétique, CEN Saclay, 91191 Gif sur Yvette Cedex, France. and Essam [12]. However, for a long time, the exponent $v$ of the two dimensional percolation was not known with enough accuracy to eliminate at least one of the two conjectured values $(v=4 / 3$ by den Nijs [13] and $v=1.3548$ by Klein et al. [14]). The situation has been clarified recently by Eschbach $e t$ al. [15] who found a good agreement with the den Nijs conjecture by increasing the accuracy of Monte Carlo renormalizations which previously seemed to support the predictions of Klein et al. [14]. Blöte et al. [16] found also a good agreement with $v=4 / 3$ by interpolating the values of $v$ for the $q$ state Potts model with $q$ close to 1 . It was then interesting to see if the direct application of the PR method to percolation which is much cheaper in computer time than Monte Carlo renormalization [15] could also support the den Nijs conjecture. In the results presented here, we find again an excellent agreement with $v=4 / 3$. The PR method works also very well in the case of lattice animals and we find accurate estimations for the exponent $v$.

The PR method is a consequence of the finite-size scaling idea due to Fisher and Barber [17]. The finitesize scaling allows to extract the critical behaviour of infinite systems from the numerical studies done on finite systems [18, 19]. Hamer and Barber [20] have reviewed recently what is known on finite-size scaling. Let us just mention here that it has been used recently to study the $X Y$ model [20], the Potts model [16, 21], 
the localization problem [22] and the roughening transition [23]. Finite-size scaling is expected to hold only for very large systems. Unfortunately, the systems one can study by computer are rather small. So the corrections to finite-size scaling are not always negligible. When one uses the PR method, due to these corrections, one finds estimations of the critical point of the exponents which depend on the size considered. The size dependence of these predictions is in most cases regular and the extrapolation of the results seems reliable. However, one finds sometimes non monotonic convergences and then the extrapolation becomes much more problematic. We discuss how assumptions on the corrections to finite-size scaling make possible the extrapolation of the results of the phenomenological renormalization.

The paper is organized as follows : in section 2, we examine the different ways of calculating critical points and critical exponents using the finite-size scaling and we recall how the phenomenological renormalization method can be derived from the finite-size scaling.

In section 3 we present the results we have obtained for bond and site percolation using the PR method.

In section 4, we extrapolate the results presented in section 3 and we explain how we can estimate the confidence interval on the extrapolated values of the threshold and of the critical exponent of the $2 \mathrm{~d}$ percolation.

In section 5, we explain how the transfer matrix method can be used to study the statistics of lattice animals. We compare (in the appendix B) the structure of the transfer matrix for lattice animals with its structure in the case of percolation. Lastly, we present the estimations of the PR method for lattice animals.

2. Finite-size scaling and phenomenological renormalization. - To simplify the discussion, we consider an infinite system which is two dimensional and "finite" systems which are infinite strips of finite width $n$. One can easily generalize what follows to cases where the infinite system has any dimension and where the "finite" systems are finite at least in one direction. Again, for simplicity, we consider that there is only one parameter $T$ (the temperature) in the problem and that the infinite system has a second order phase transition at a critical temperature $T_{\mathrm{c}}$. For a given physical quantity $Q$, the typical situation is that $Q$ is singular at $T_{\mathrm{c}}$ in the infinite system

$$
\left[Q_{\infty}(T) \sim\left|T-T_{\mathrm{c}}\right|^{-\omega}\right]
$$

whereas it is regular in the finite systems $\left[Q_{n}(T)\right.$ has no singularity]. When $n$ increases, the singularity of $Q_{\infty}(T)$ starts to develop [for example, if $Q_{\infty}(T)$ diverges at $T_{c}, Q_{n}(T)$ has a maximum which becomes sharper and sharper]. The content of the finite-size scaling hypothesis $[17,20]$ is to assume the existence of scaling functions $F_{Q}$ such that :

$$
Q_{n}(T) \simeq Q_{\infty}(T) F_{Q}\left(n / \xi_{\infty}(T)\right)
$$

where $\xi_{\infty}(T)$ is the correlation length in the infinite system. This relation is expected to be valid when the size $n$ of the finite system is large and when $T$ approaches $T_{\mathrm{c}}$. However Brezin [24] has shown recently that relation (1) fails above the upper critical dimensionality. As far as $n$ is finite, $Q_{n}(T)$ is a regular function of $T$. This means that $F_{Q}$ compensates the singularities of $Q_{\infty}(T)$ and of $\xi_{\infty}(T):$ if

$$
\begin{aligned}
& Q_{\infty}(T) \sim\left|T-T_{\mathrm{c}}\right|^{-\omega} \\
& \xi_{\infty}(T) \sim\left|T-T_{\mathrm{c}}\right|^{-v}
\end{aligned}
$$

then $F_{Q}$ must behave like :

$$
F_{Q}(z) \sim z^{\omega / v} \text { for } z \rightarrow 0 .
$$

It follows that at $T_{\mathrm{c}}, Q_{n}$ depends on $n$ as a power law :

$$
Q_{n}\left(T_{\mathrm{c}}\right) \sim n^{\omega / v} \text { for large } n .
$$

If $Q^{(p)}(T)$ is the $p$ th derivative of $Q(T), Q_{\infty}^{(p)}(T)$ is also singular at $T_{\mathrm{c}}$

$$
\frac{\mathrm{d}^{p}}{\mathrm{~d} T^{p}} Q_{\infty}(T)=Q_{\infty}^{(p)}(T) \sim\left|T-T_{\mathrm{c}}\right|^{-\omega-p}
$$

and therefore

$$
Q_{n}^{(p)}\left(T_{\mathrm{c}}\right) \sim n^{(\omega+p) / v} .
$$

Because $Q_{n}(T)$ is a regular function of $T$, it has a Taylor expansion around $T_{\mathrm{c}}$

$$
Q_{n}(T)=\sum_{p=0}^{\infty} Q_{n}^{(p)}\left(T_{\mathrm{c}}\right) \frac{\left(T-T_{\mathrm{c}}\right)^{p}}{p !} .
$$

And using (7) we find that $Q_{n}(T)$ can be expressed as

$$
Q_{n}(T) \sim n^{\omega / v} G_{Q}\left(n^{1 / v}\left(T-T_{\mathrm{c}}\right)\right)
$$

where $G_{Q}$ is a scaling function which is regular around $T_{\mathrm{c}}$. It is interesting to notice that, usually, the functions $F_{Q}$ and $G_{Q}$ depend not only on the physical quantity $Q$ but also on the boundary conditions of the finite system [19]. Recently dos Santos and Stinchcombe [25] have also extended the ideas of finite-size scaling to cases where there are more than one parameter.

At this stage, we see that finite-size scaling gives us many possibilities to calculate the critical properties of a given model. According to what is known on the problem, to the accuracy one can reach in the calculation of $Q_{n}(T)$, to the available computer time, etc., one chooses one of these possibilities. We recall now what are the usual methods using the finite-size scaling.

A first method [1] (that we shall use in this paper) is the phenomenological renormalization. Let us recall briefly its principle. One starts by calculating the correlation length $\xi_{n}(T)$ for strips of width $n$ using a 
transfer matrix. Then one writes the fundamental equation of the PR method

$$
\frac{\xi_{n}(T)}{\xi_{m}\left(T^{\prime}\right)}=\frac{n}{m} .
$$

The main hypothesis of the PR method is to assume that the relation (10) between $T$ and $T^{\prime}$ depends only on the ratio $\mathrm{n} / \mathrm{m}$. Therefore one has

$$
\frac{\xi_{n}(T)}{\xi_{m}\left(T^{\prime}\right)}=\frac{n}{m}=\frac{\xi_{\infty}(T)}{\xi_{\infty}\left(T^{\prime}\right)} .
$$

To justify this relation, one could argue that the strip of width $n$ at temperature $T$ is related by a scaling transformation to a strip of width $m$ at temperature $T^{\prime}$. This transformation is a contraction of ratio $n / m$ of the width of the strip and of the correlation length $\xi_{n}$ which is the characteristic length along the strip.

However it is easy to show that (11) is a direct consequence of the finite-size scaling hypothesis (1). Consider two temperatures $T$ and $T^{\prime}$ such that

$$
\frac{\xi_{\infty}(T)}{\xi_{\infty}\left(T^{\prime}\right)}=\lambda
$$

For any choice of the scaling factor $\lambda$, equation (12) gives us a relation between $T$ and $T^{\prime}$. Using expression (1) we can write the ratio $Q_{n}(T) / Q_{m}\left(T^{\prime}\right)$ as

$$
\frac{Q_{n}(T)}{Q_{m}\left(T^{\prime}\right)}=\frac{Q_{\infty}(T)}{Q_{\infty}\left(T^{\prime}\right)} \frac{F_{Q}\left[n / \xi_{\infty}(T)\right]}{F_{Q}\left[m / \xi_{\infty}\left(T^{\prime}\right)\right]} .
$$

By choosing $\lambda=n / m$, the function $F_{Q}$ is eliminated in (13) because its two arguments are identical. Therefore one has in this case

$$
\frac{Q_{n}(T)}{Q_{m}\left(T^{\prime}\right)}=\frac{Q_{\infty}(T)}{Q_{\infty}\left(T^{\prime}\right)} .
$$

By applying this result to the correlation length itself one recovers (11)

$$
\frac{\xi_{\infty}(T)}{\xi_{\infty}\left(T^{\prime}\right)}=\frac{n}{m}=\frac{\xi_{n}(T)}{\xi_{m}\left(T^{\prime}\right)} .
$$

The simplest way to calculate $T_{\mathrm{c}}(n, m)$ using the PR method is to solve the following equation :

$$
\frac{\xi_{n}\left(T_{\mathrm{c}}\right)}{n}=\frac{\xi_{m}\left(T_{\mathrm{c}}\right)}{m} .
$$

Once $T_{\mathrm{c}}(n, m)$ is known, one can easily calculate the critical exponents from equation (14). For example $v$ is given by

$$
1+\frac{1}{v}=\frac{\log \left[\frac{\mathrm{d} \xi_{n}}{\mathrm{~d} T}\left(T_{\mathrm{c}}\right) / \frac{\mathrm{d} \xi_{m}}{\mathrm{~d} T}\left(T_{\mathrm{c}}\right)\right]}{\log [n / m]} .
$$

In some cases [16, 21], the simplest quantity $Q$ to calculate is not the correlation length $\xi$. Using relation (5), one can find $T_{\mathrm{c}}$ and the ratio $\omega / v$ by searching the temperature where $Q_{n}$ behaves as a power law of $n$. One chooses three widths $n, m$ and $p$ and one calculates $T_{\mathrm{c}}(n, m, p)$ as the solution of

$$
\frac{\log \left[Q_{m}\left(T_{\mathrm{c}}\right) / Q_{n}\left(T_{\mathrm{c}}\right)\right]}{\log \left[Q_{p}\left(T_{\mathrm{c}}\right) / Q_{n}\left(T_{\mathrm{c}}\right)\right]}=\frac{\log [m / n]}{\log [p / n]} .
$$

Once $T_{\mathrm{c}}(n, m, p)$ is known, the ratio $\omega / v$ is easily obtained by

$$
\frac{\omega}{v}=\frac{\log \left[Q_{m}\left(T_{\mathrm{c}}\right) / Q_{n}\left(T_{\mathrm{c}}\right)\right]}{\log [m / n]} .
$$

Of course, one can use again equation (19) by replacing $Q$ by $Q^{(p)}$ and $\omega$ by $\omega+p$. Thus one can calculate $\omega$ and $v$.

Another method consists in using equation (9). One calculates $Q_{n}(T)$ for several sizes $n$. Then one tries to determine what is the best choice of $\omega, v$ and $T_{\mathrm{c}}$ which allows to superpose the curves $n^{-\omega / v} Q_{n}(T)$ as a function of $n^{1 / v}\left(T-T_{\mathrm{c}}\right)$. This approach is more suitable to study disordered systems (random magnets, localization [22] ...). In these problems, the calculated quantities $Q_{n}(T)$ are obtained by products of random matrices and therefore affected by statistical errors which do not allow to use formulae (17) or (19).

Of course the main difficulty is that the finite-size scaling is valid only for large $n$ and that the numerical calculations can be done only for small $n$. Therefore the corrections to finite-size scaling cannot be neglected. In section 4 we shall show that reasonable assumptions on the corrections to finite-size scaling allow to give rather accurate predictions for the critical points and the critical exponents.

3. Application to percolation. - In this section we present the results we have obtained for the two dimensional percolation. We calculated the correlation lengths of strips as function of the probability $p$ of present bonds or sites in the same way as in reference [7]. We chose two kinds of boundary conditions in the direction perpendicular to the strip : the periodic boundary conditions and the helical boundary conditions [9]. In the case of periodic boundary conditions a strip of width $n$ means that a site can be labelled by two integers $(i, j)$ with $-\infty<i<+\infty$ and $1 \leqslant j \leqslant n$ and that the four neighbours of this site are $(i \pm 1, j)$ and $(i, j \pm 1+k n)$ [where $k$ is the integer such that $1 \leqslant j \pm 1+k n \leqslant n]$. In the case of helical boundary conditions, a site can be labelled by a single integer $i(-\infty<i<+\infty)$ and its four nearest neighbours are the sites $i \pm 1$ and $i \pm n$.

The most difficult part of the calculation was the construction of the transfer matrix for large widths. We used a construction similar to the one described 
Table I. - Results of the phenomenological renormalization for site percolation. We have calculated the correlation lengths $\xi_{n}$ with two kinds of boundary conditions on the strips : periodic boundary conditions and helical boundary conditions. For each choice of $n$ and $m$, the estimations of $p_{c}$ and $v$ were obtained from equations (16) and (17). The extrapolations were done by assuming a power law convergence $\left(n^{-x}\right)$. The results are in good agreement with previous works : [a] = Ref. [26], [b] = Ref. [13], [c] = Ref. [16].

\begin{tabular}{|c|c|c|c|c|c|}
\hline & & \multicolumn{4}{|c|}{ SITE PERCOLATION } \\
\hline & & \multicolumn{2}{|c|}{ Periodic bound. cond. } & \multicolumn{2}{|c|}{ Helical bound cond. } \\
\hline$n$ & $m$ & $p_{\mathrm{c}}$ & $v$ & $p_{\mathrm{c}}$ & $v$ \\
\hline 2 & 1 & 0.73389 & 1.5604 & 0.62712 & 1.5712 \\
\hline 3 & 2 & 0.58214 & 1.4932 & 0.65594 & 1.5566 \\
\hline 4 & 3 & 0.59096 & 1.4718 & 0.61384 & 1.4328 \\
\hline 5 & 4 & 0.58870 & 1.4139 & 0.60439 & 1.4111 \\
\hline 6 & 5 & 0.58992 & 1.3880 & 0.59918 & 1.3880 \\
\hline 7 & 6 & 0.59068 & 1.3717 & 0.59667 & 1.3741 \\
\hline 8 & 7 & 0.59127 & 1.3616 & 0.59529 & 1.3645 \\
\hline 9 & 8 & 0.59188 & 1.3548 & 0.59449 & 1.3578 \\
\hline 10 & 9 & & & 0.59399 & 1.3530 \\
\hline \multicolumn{2}{|c|}{ Expected values } & $0.5931 \pm 0.0006[\mathrm{a}]$ & $\begin{array}{l}1.3333[\mathrm{~b}] \\
1.333 \pm 0.002[\mathrm{c}]\end{array}$ & $0.5931 \pm 0.0006$ & 1.3333 \\
\hline \multicolumn{2}{|c|}{ Extrapolation } & & $1.330 \pm 0.005$ & $0.5927 \pm 0.0002$ & $1.330 \pm 0.005$ \\
\hline \multicolumn{2}{|c|}{$x$} & & $2.1 \pm 0.4$ & $3.1 \pm 0.4$ & $1.8 \pm 0.4$ \\
\hline
\end{tabular}

Table II. - The same as table I for bond percolation.

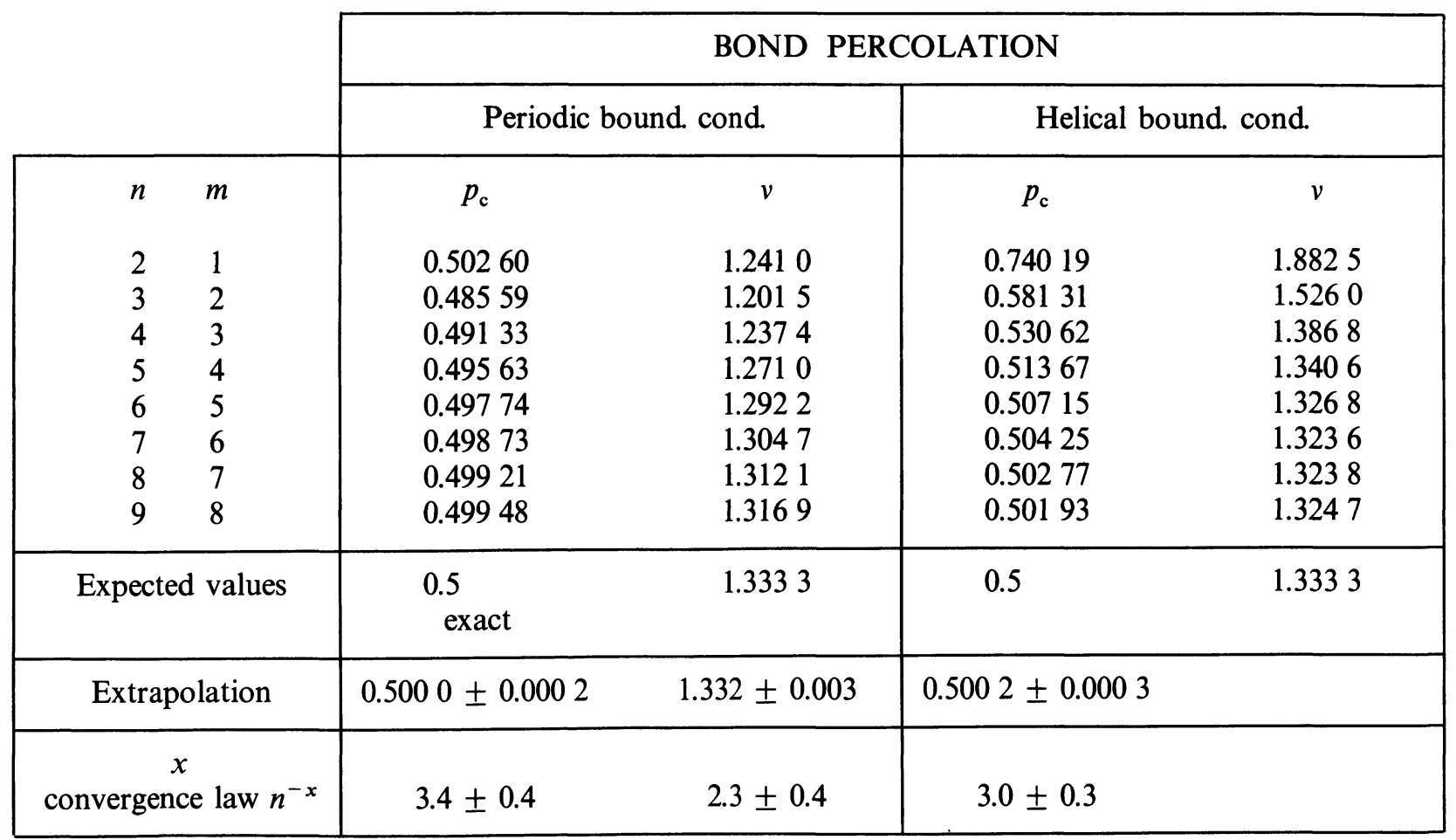


in reference [16] for the Potts model. The only difference is that the configurations of connected sites are not exactly the same because we calculate here the correlation length instead of the free energy. Once the transfer matrix is constructed, the largest elgenvalue $[7,27]$ which gives the correlation length can be easily obtained by multiplying the transfer matrix. The estimations of $p_{\mathrm{c}}$ and $v$ given in tables I and II were then found by solving equation (16) and from equation (17) for several choices of $n$ and $m$. As we shall discuss it in section 4 , the best choice for $n$ and $m$ is $m=n-1$ and $n$ as large as possible.

For site percolation (Table I) as well as for bond percolation (Table II) the values found for $p_{\mathrm{c}}$ or $v$ are close to their expected values. The convergence is often regular and this will allow us to give extrapolated values with small confidence intervals. However in some cases the convergence is not monotonic ( $p_{\mathrm{c}}$ for site percolation with periodic boundary conditions or $v$ for bond percolation with helical boundary conditions). In the next section, we explain the extrapolation method we have used.

4. Extrapolation. - The convergence law of the results of the PR method is related to the corrections to the finite-size scaling. As we saw it in section 2, at the critical temperature, the correlation length is linear in $n\left(\xi_{n}\left(T_{c}\right) \sim n\right)$ and its derivatives are power laws. By analogy with the Ising model (see the appen$\operatorname{dix} \mathrm{A}$ ), it seems reasonable to assume that the corrections to the finite-size scaling are power laws (we even assume that it is the same power law for the correlation length and its first derivatives)

$$
\begin{aligned}
\xi_{n}\left(T_{\mathrm{c}}\right) & =A_{0} n\left(1+A_{1} n^{-a}+\cdots\right) \\
\frac{\mathrm{d} \xi_{n}}{\mathrm{~d} T}\left(T_{\mathrm{c}}\right) & =B_{0} n^{1+\frac{1}{v}}\left(1+B_{1} n^{-a}+\cdots\right) \\
\frac{\mathrm{d}^{2} \xi_{n}}{\mathrm{~d} T^{2}}\left(T_{\mathrm{c}}\right) & =C_{0} n^{1+\frac{2}{v}}(1+\cdots) .
\end{aligned}
$$

For large $n$ and $m$, the estimation $T_{\mathrm{c}}(n, m)$ which can be found by solving equation (16) is :

$$
T_{\mathrm{c}}(n, m) \simeq T_{\mathrm{c}}-\frac{A_{0} A_{1}}{B_{0}} \frac{n^{-a}-m^{-a}}{n^{1 / v}-m^{1 / v}}+\cdots
$$

Using this estimation $T_{\mathrm{c}}(n, m)$, one can calculate the estimation $v(n, m)$ from equation (17)

$v(n, m) \sim v-v^{2}\left(B_{1}-\frac{A_{0} C_{0}}{B_{0}^{2}} A_{1}\right) \frac{n^{-a}-m^{-a}}{\log (n / m)}+\cdots$.

The formulae (21) and (22) give the relation between the corrections to finite-size scaling and the results of the PR method. For a given width $n$, it is easy to show that the convergence is improved by choosing $m=n-1$. For this choice one finds that

$$
\begin{aligned}
T_{\mathrm{c}}(n, n-1)-T_{\mathrm{c}} & \sim a v \frac{A_{0} A_{1}}{B_{0}} n^{-a-\frac{1}{v}} \\
v(n, n-1)-v & \sim v^{2} a\left(B_{1}-\frac{A_{0} C_{0}}{B_{0}^{2}} A_{1}\right) n^{-a} .
\end{aligned}
$$

The extrapolated values given in tables I and II were obtained by assuming a power law convergence like in reference [4]. We have plotted

$$
\log \left(T_{\mathrm{c}}(n, n-1)-T\right)
$$

as a function of $\log n$ for several choices of $T$. The extrapolated value and the confidence intervals were obtained by looking for the values of $T$ for which the curvature was small. In several cases the convergence was monotonic and using this procedure, we could find the extrapolated values, the confidence intervals and the exponent $x$ of the power law convergence. In other cases $\left(p_{\mathrm{c}}\right.$ for site percolation with periodic boundary conditions or $v$ for bond percolation with helical boundary conditions) the convergence was not monotonic and we could not find a simple method of extrapolating our results. This non monotonic convergence is probably due to higher order corrections to the finite-size scaling.

The coefficients $A_{0}, B_{0}, C_{0} \ldots$ have probably a physical interpretation. For the $X Y$ model in 2 dimension, it has been shown $[22,28]$ that $A_{0}$ was related to the critical exponent $\eta$ at least at low temperature

$$
A_{0}=\frac{1}{\pi \eta}
$$

This relatior remains probably true for several isotropic systems in dimension 2. For the Ising, model $\eta=1 / 4$ and $A_{0}=4 / \pi$ (appendix A). From references [13] and [29], the conjectured prediction of $\eta$ for the $q$ state Potts model $(q<4)$ is

$$
\eta=\frac{1-4 p^{2}}{4(1-p)} \text { with } \quad \cos \pi p=\frac{\sqrt{q}}{2} .
$$

For bond percolation on strips with periodic boundary conditions we have calculated the correlation lengths at $p=1 / 2$. We found the linear behaviour of $\xi_{n}(1 / 2)$ and an estimation of $A_{0}$ :

$$
A_{0}=1.5277 \pm 0.0005 \text {. }
$$

This estimation is in good agreement with relation (24) $(1 / \pi \eta=1.5279)$. It is possible that (24) remains true for a large class of $2 \mathrm{~d}$ isotropic models [30].

5. Application to lattice animals. - The problem of lattice animals [31, 32] lies between percolation and self avoiding walks. One considers $s$ connected sites on a given lattice. One expects that the number 
Table III. - Results of the phenomenological renormalization for lattice animals. They agree well with previous

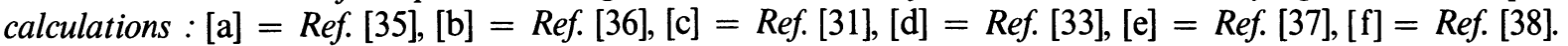

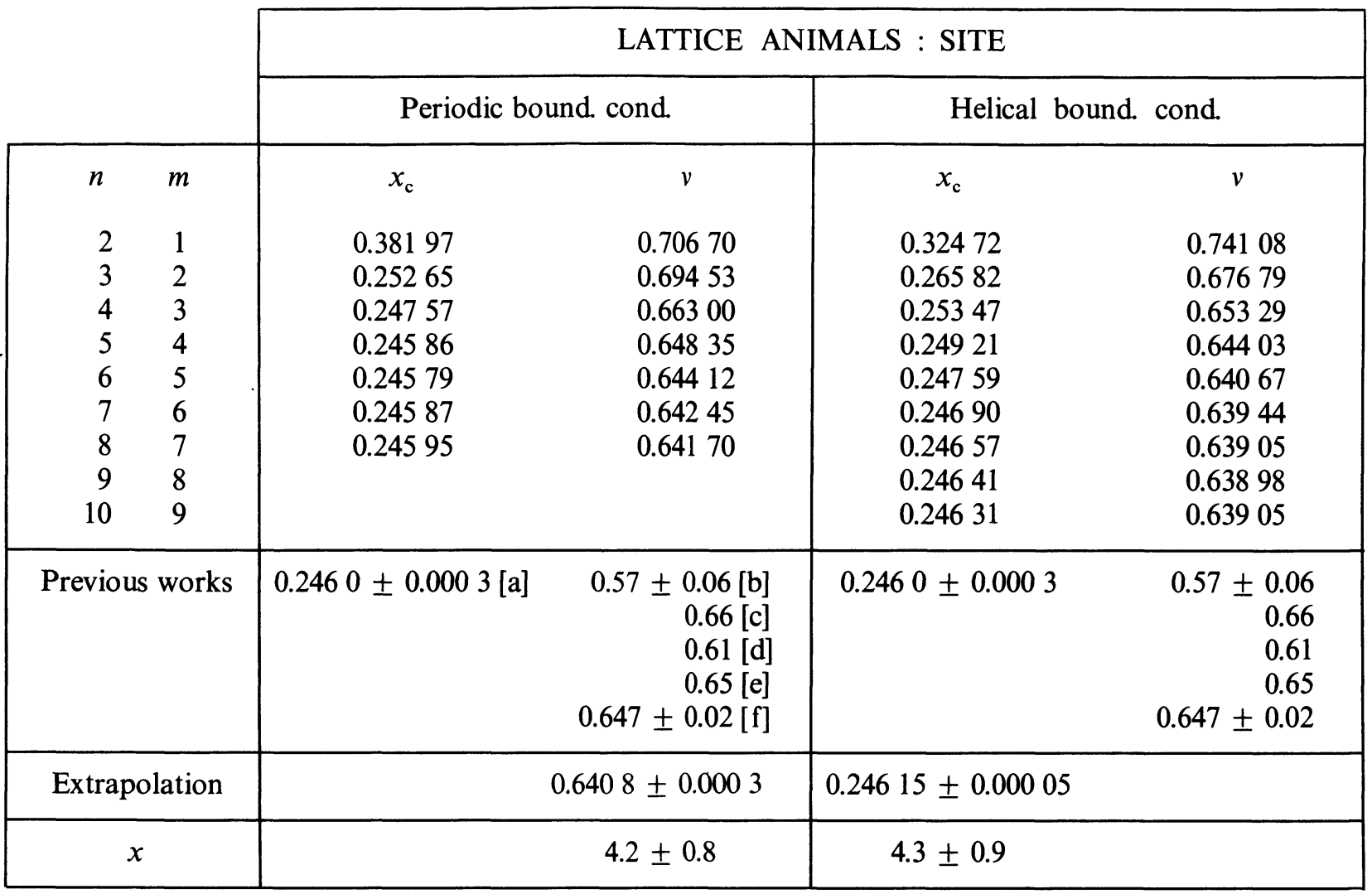

$\mathcal{N}_{s}$ of different configurations of these $s$ connected sites behaves for large $s$ like :

$$
\mathcal{N}_{s} \sim s^{-\theta} \mu^{s}
$$

where $\mu$ is a constant which depends on the details of the lattice and $\theta$ is a universal exponent which depends only on the dimension $d$ of space. The average size $R$ of these configurations is related to the number of sites $s$ by another universal exponent $v$

$$
R \sim s^{v} .
$$

Recently Parisi and Sourlas [33] have shown that $\theta$ and $v$ in dimension $d$ are not independent $\left(v=\frac{\theta-1}{d-2}\right)$ and are related to the exponent of the Lee and Yang singularity in dimension $d-2$. However their relation is useless to calculate the exponent $v$ in dimension 2 .

We have calculated this exponent $v$ by the PR method. To do so it is necessary to define a correlation length for the lattice animals problem. The simplest way is to consider generating functions. If $\mathcal{N}_{s}(0, \mathbf{R})$ is the number of animals of $s$ sites which occupy both sites 0 and $\mathbf{R}$, one can define the generating function $G_{0 \mathbf{R}}(x)$ of these numbers :

$$
G_{\mathrm{OR}}(x)=\sum_{s} x^{s} \mathcal{N}_{s}(0, \mathbf{R})
$$

The parameter $x$ plays the same role as the probability $p$ in percolation problems or the temperature $T$ in spin problems. Its critical value $x_{\mathrm{c}}$ is equal to $\mu^{-1}$. By analogy with critical phenomena, the function $G_{\mathbf{O R}}(x)$ behaves as a correlation function for $x<x_{\mathbf{c}}$

$$
G_{0 \mathbf{R}}(x) \sim \exp (-R / \xi(x)) \text { for } R \rightarrow \infty .
$$

It can be shown that

$$
\sum_{\mathbf{R}} G_{0 \mathbf{R}}(x) \sim(1-\mu x)^{\theta-2}
$$

and

$$
\xi(x) \sim(1-\mu x)^{-v}
$$

where $\theta$ and $v$ are defined by equations (27) and (28).

By applying to lattice animals the ideas yet developed for percolation [7], self avoiding walks $[8,34]$ and the Potts model [16], we have calculated the correlation length $\xi_{n}(x)$ for lattice animals on strips of width $n$ (appendix B).

Using these correlation lengths $\xi_{n}(x)$, we have calculated the estimations

$$
x_{\mathrm{c}}(n, n-1) \text { and } v(n, n-1)
$$

for several choices of $n$ and for periodic and helical boundary conditions (Table III). The extrapolation was done in the same way as explained in section 4. 
Here again we could not extrapolate our results when the convergence was not monotonic ( $x_{\mathrm{c}}$ for periodic boundary conditions, $v$ for helical boundary conditions). Our results are in good agreement with previous works $[31,33,35,36,37]$. Even when the convergence is not monotonic, the estimations are very close to the extrapolated values that we give.

6. Conclusion. - We have reported here the results of our calculations on $2 \mathrm{~d}$ percolation and $2 \mathrm{~d}$ lattice animals using the phenomenological renormalization. The method works very well for both problems and leads to accurate predictions on the critical point and the exponent $v$.

We think that the PR is a simple method to study a large class of models in statistical mechanics. Its main advantages are that :

- Only one parameter is renormalized;

- It gives satisfactory results with reasonable calculations;

- The results are usually improved by increasing the width of strips and the convergence seems to be rather rapid (a power law);

- For each width the estimations are obtained without error bars and for this reason it is easier to estimate the confidence intervals on the extrapolated values;

- In principle, the method can be applied to models (like for example frustrated models) where the order parameter is not known a priori. The reason is that the correlation length can be calculated from the two largest eigenvalues of the transfer matrix without knowing what correlation function decreases with this correlation length.

However the method presents some difficulties. In particular it is hard to study models in dimension 3 because the size of the transfer matrices becomes too large. Moreover, the sequence of calculated values has sometimes a non-monotonic convergence and does not allow to extract accurate estimations. We must emphasize that these problems of convergence can be completely masked in Monte Carlo simulations by statistical errors.

Acknowledgments. - We are grateful to E. Brezin, J. M. Luck, J. L. Pichard, G. Sarma and D. Stauffer for stimulating discussions.

Appendix A. - In this appendix we give the analytic expression of the large $n$ expansion of the correlation length and of its first derivatives at $T_{\mathrm{c}}$ for strips of Ising spins with periodic boundary conditions. The corrections to finite-size scaling are power laws and it is possible to calculate analytically the convergence law of $K_{\mathrm{c}}(n, m)$ and $v(n, m)$.

The correlation lengths for strips of width $n$ with periodic boundary conditions are known exactly (they are recalled in reference [1]). If the nearest neighbour interaction is $K$ :

$$
\mathscr{H}=-K \sum_{\langle i j\rangle} \sigma_{i} \sigma_{j}
$$

then the correlation length $\xi_{n}(K)$ is given by

$$
\begin{aligned}
\frac{1}{\xi_{n}(K)}=\frac{1}{2}\left[\left(\gamma_{1}+\gamma_{3}+\cdots+\gamma_{2 n-1}\right)-\right. \\
\left.-\left(\gamma_{0}+\gamma_{2}+\cdots+\gamma_{2 n-2}\right)\right]
\end{aligned}
$$

where the $\gamma_{r}$ are given by

$$
\cosh \gamma_{r}=\frac{\cosh ^{2} 2 K}{\sinh 2 K}-\cos \frac{r \pi}{n} \text {. }
$$

The critical value $K_{\mathrm{c}}$ is known :

$$
K_{\mathrm{c}}=\frac{1}{2} \log (1+\sqrt{2}) \text {. }
$$

From (A.2) and (A.3), one can find for the Ising model the values of $A_{0}, A_{1}, B_{0}, B_{1}, C_{0}$ and $a$ of equation (20) :

$$
\begin{aligned}
\xi_{n} & \sim \frac{4}{\pi} n\left(1-\frac{\pi^{2}}{24 n^{2}}+\cdots\right) \\
\frac{\mathrm{d} \xi_{n}}{\mathrm{~d} K} & \sim \frac{32}{\pi^{2}} n^{2}\left(1-\frac{\pi^{2}}{12 n^{2}}+\cdots\right) \\
\frac{\mathrm{d}^{2} \xi_{n}}{\mathrm{~d} K^{2}} & =\frac{128}{\pi^{3}} n^{3}(4-2 \log 2)+\cdots
\end{aligned}
$$

Then from equations (21) and (22), one finds that :

$$
\begin{aligned}
K_{\mathrm{c}}(n, m) & =\frac{1}{2} \log (1+\sqrt{2})-\frac{\pi^{3}}{192} \frac{\lambda(\lambda+1)}{n^{3}}+\cdots \\
v(n, m) & =1-\frac{\pi^{2} \log 2}{24 n^{2}} \frac{\lambda^{2}-1}{\log \lambda}+\cdots
\end{aligned}
$$

where $\lambda=n / m$. One can notice that one can minimize these corrections by choosing $\lambda$ as close as possible to 1 , i.e. $n=m-1$.

Appendix B. - In this appendix, we give as an example the transfer matrix for site percolation and lattice animals on a strip of width 4 with periodic boundary conditions.

TRANSFER MATRIX FOR SITE PERCOLATION. - One consider a strip of 4 lines with periodic boundary conditions. We call $p$ the probability that a site is occupied and $q=1-p$ the probability that a site is empty. The probability $P_{N}(p)$ that the first and the $N$ th column are connected decreases exponentially

$$
P_{N}(p) \sim \mathrm{e}^{-N / \xi(p)}
$$




$\begin{array}{cccccc}- & 0 & 0 & 0 & 0 & 0 \\ - & 0 & 0 & 0 & 0 & 0 \\ 0 & 0 & 0 & 0 & x & 0 \\ - & 0 & O & 0 & 0 & 0 \\ A & \text { B } & \text { C } & \text { D } & \text { E } & \text { F }\end{array}$

Site occupied and connected.
Site empty
$x \quad$ Site occupied but not connected.

Fig. 1. - The 6 configurations of a strip of width $n=4$ with periodic boundary conditions, for site percolation. We call a site connected if it has been connected to column 1 by the portion of the strip included between column 1 and column $N$.

To calculate the correlation length $\xi(p)$ we follow the same method as in reference [7]. First we have to list all the possible configurations at column $N$ (see Fig. 1). Due to the periodic boundary conditions, it remains only 6 configurations. Then we have to relate the probabilities $A_{N}, B_{N}, C_{N}, D_{N}, E_{N}, F_{N}$ of these configurations at column $N$ to their probabilities at column $N+1$

$$
\begin{aligned}
& A_{N+1}=p^{4}\left(A_{N}+B_{N}+C_{N}+D_{N}+E_{N}+F_{N}\right) \\
& B_{N+1}=p^{3} q\left(4 A_{N}+4 B_{N}+4 C_{N}+4 D_{N}+3 E_{N}+3 F_{N}\right) \\
& C_{N+1}=p^{2} q^{2}\left(4 A_{N}+4 B_{N}+3 C_{N}+4 D_{N}+2 E_{N}+2 F_{N}\right) \\
& D_{N+1}=p^{2} q^{2}\left(2 A_{N}+B_{N}+D_{N}\right) \\
& E_{N+1}=p^{2} q^{2}\left(B_{N}+2 C_{N}+E_{N}+F_{N}\right) \\
& F_{N+1}=p q^{3}\left(4 A_{N}+3 B_{N}+2 C_{N}+2 D_{N}+E_{N}+F_{N}\right) .
\end{aligned}
$$

If $\lambda_{1}(p)$ is the largest eigenvalue of this matrix, it is clear that each of these probabilities behaves like $\left[\lambda_{1}(p)\right]^{N}$ for large $N$. Therefore one has

$$
\lambda_{1}(p)=\exp \left[-\frac{1}{\xi(p)}\right]
$$

TRANSFER MATRIX FOR SITE LATTICE ANIMALS. - For percolation, the important quantity was the probability $P_{N}(p)$ that the column $N$ is connected to column 1 . For lattice animals, the important quantity is $G_{N}(x)$

$$
G_{N}(x)=\sum_{s} x^{s} \mathcal{N}_{s}(1, N)
$$

where $\mathcal{N}_{s}(1, N)$ is the number of animals of $s$ sites connecting column 1 to column $N$. As in percolation, we have to list all the possible configurations at column $N$. We find exactly the same configurations as in percolation (Fig. 1). Each configuration corresponds to a part of sum (B.4). Configuration A corresponds to the sum $A_{N}$ over all the animals which connect columns 1 and $N$ and occupy the 4 sites of column $N$; configuration $\mathrm{E}$ corresponds to the sum $E_{N}$ over all the animals which connect columns 1 and $N$ and occupy 2 sites of column $N$ (one of these 2 sites has not been connected to column 1 by the portion of strip included between columns 1 and $N$ ). As in percolation, it is easy to write the linear relations between

and

$$
\left\{A_{N}, B_{N}, \ldots, F_{N}\right\}
$$

$A_{N+1}=x^{4}\left(A_{N}+B_{N}+C_{N}+D_{N}+E_{N}+F_{N}\right)$

$B_{N+1}=x^{3}\left(4 A_{N}+4 B_{N}+4 C_{N}+4 D_{N}+2 E_{N}+3 F_{N}\right)$

$C_{N+1}=x^{2}\left(4 A_{N}+4 B_{N}+3 C_{N}+4 D_{N}+2 F_{N}\right)$

$D_{N+1}=x^{2}\left(2 A_{N}+B_{N}+D_{N}\right)$

$E_{N+1}=x^{2}\left(B_{N}+2 C_{N}+E_{N}+F_{N}\right)$

$F_{N+1}=x\left(4 A_{N}+3 B_{N}+2 C_{N}+2 D_{N}+F_{N}\right)$.

Here again, for large $N, G_{N}(x)$ behaves like $\left[\lambda_{1}(x)\right]^{N}$ where $\lambda_{1}(x)$ is the largest eigenvalue of this matrix. Therefore the correlation length $\xi(x)$ can be calculated by

$$
\lambda_{1}(x)=\exp \left[-\frac{1}{\xi(x)}\right]
$$

We see the resemblance between the transfer matrix (B. 2) for percolation and the transfer matrix (B.5) for lattice animals. The only difference, at first sight, is that $p$ is replaced by $x$ and $q$ by 1 . However the fact that the $s$ sites of an animal are connected imposed constraints. This can be seen by comparing the coefficients of $E_{N}$ in the two matrices. The site of configuration $E$ which is occupied but not connected plays no role in percolation. On the contrary, for lattice animals, this site must be connected by the portion of the strip which follows the column $N$. This is why configuration $\mathrm{E}$ at column $N$ cannot be followed by configuration $\mathrm{F}$ at column $N+1$ because this site would never be connected.

\section{References}

[1] Nightingale, M. P., Physica 83A (1976) 561.

[2] Nightingale, M. P., Proc. Kon. Ned. Ak. Wet. B 82 (1979) 235.

SNEDDON, L., J. Phys. C 11 (1978) 2823.

[3] Sneddon, L., J. Phys. C 12 (1979) 3051.
[4] Racz, Z., Phys. Rev. B 21 (1980) 4012.

KINZEL, W. and SCHICK, M., preprint (1981).

[5] Sneddon, L. and Stinchcombe, R. B., J. Phys. C 12 (1979) 3761.

[6] Uzelac, K. and Jullien, R., J. Phys. A 14 (1981) L-151. 
[7] Derrida, B. and Vannimenus, J., J. Physique-Lett. 41 (1980) L-473.

[8] Derrida, B., J. Phys. A 14 (1981) L-5.

[9] Kinzel, W. and Yeomans, J. M., J. Phys. A 14 (1981) L-163.

[10] KirkPatricK, S., Les Houches 1978, eds. Balian R., Maynard R. and Toulouse G. (North Holland).

[11] Stauffer, D., Phys. Rep. 54 (1979) 1.

[12] Essam, J. W., Rep. Prog. Phys. 43 (1980) 833.

[13] Den NiJs, M. P. M., J. Phys. A 12 (1979) 1857.

[14] Klein, W., Stanley, H. E., Reynolds, P. J. and Coniglio, A., Phys. Rev. Lett. 41 (1978) 1145.

[15] Esbach, P. D., Stauffer, D. and Herrmann, H. J., Phys. Rev. B 23 (1981) 422.

[16] Blöte, H. W. J., Nightingale, M. P. and Derrida, B., J. Phys. A 14 (1981) L-45.

[17] Fisher, M. E., in Critical Phenomena, Proc. Int. School of Physics « Enrico Fermi », Varenna 1970, Course $n^{\circ}$ 51, ed. M. S. Green (N.Y. Academic) 1971.

Fisher, M. E. and BARBER, N. M., Phys. Rev. Lett. 28 (1972) 1516.

SuzukI, M., Prog. Theor. Phys. 58 (1977) 1142.

[18] Landau, D. P., Phys. Rev. B 13 (1976) 2997.

[19] Heermann, D. W. and Stauffer, D., Z. Phys. B 40 (1980) 133.

[20] Hamer, C. J. and Barber, N. B., J. Phys. A 13 (1980) L-169; J. Phys. A 14 (1981) 241, 259.

[21] Nightingale, M. P. and Blöte, H. W. J., Physica 104A (1980) 352.

[22] Pichard, J. L. and Sarma, G., J. Phys. C 14 (1981) L-127; J. Phys. C 14 (1981) L-617.

[23] Luck, J. M., J. Physique-Lett. 42 (1981) L-271.

[24] BREZIN, E., preprint (1981).
[25] Dos Santos, R. R. and Stinchcombe, R. B., preprint (1981).

[26] Reynolds, P. J., Stanley, H. E. and Klein, W., Phys. Rev. B 21 (1980) 1223.

[27] For most problems of statistical mechanics, one needs the two largest eigenvalues of the transfer matrix to calculate the correlation length. However, as it was yet said in reference [7], for percolation, the correlation length is related only to the largest eigenvalue of the transfer matrix.

[28] LUCK, J. M., private communication.

[29] Nienhuis, B., Riedel, E. K. and Schick, M., J. Phys. A 13 (1980) L-189.

[30] We have tested numerically the relation $A_{0}=1 / \pi \eta$ for the $q$ state Potts model in dimension 2. It seems to be true for all values of $q<4$. It is particularly well verified for small values of $q(q \rightarrow 0)$ because the linear behaviour of correlation lengths at $T_{\mathrm{c}}$ occurs for small widths.

[31] Stauffer, D., Phys. Rev. Lett. 41 (1978) 1333.

[32] Lubensky, T. C. and Isaacson, J., Phys. Rev. Lett. 41 (1978) 829.

[33] Parisi, G. and Sourlas, N., Phys. Rev. Lett. 46 (1981) 871.

[34] Klein, D. J., J. Stat. Phys. 23 (1980) 561.

[35] Guttmann, A. J. and Gaunt, D. S., J. Phys. A 11 (1978) 949.

[36] Redner, S., J. Phys. A 12 (1979) L-239.

[37] Holl, K., to be published;

STAUFFER, D., Intern. Conf. on Disordered Systems to appear in Springer Lecture Notes in Physics (1981).

[38] Family, F., private communication. 